\title{
Consensus Algorithms for Distributed Spectrum Sensing Based on Goodness of Fit Test in Cognitive Radio Networks
}

\author{
Djamel TEGUIG, Bart SCHEERS, Vincent LE NIR \\ Department CISS \\ Royal Military Academy \\ Brussels, Belgium \\ Email: [djamel.teguig, bart.scheers,vincent.lenir]@rma.ac.be
}

\author{
Franois HORLIN \\ OPERA-Wireless Communication Group \\ Universit Libre de Bruxelles \\ Brussels, Belgium \\ Email: fhorlin@ulb.ac.be
}

\begin{abstract}
In this paper, we study a consensus algorithm for distributed spectrum sensing (DSS) in cognitive radio networks (CRN) integrating a Goodness of Fit based spectrum sensing scheme. Existing work in this area often applies energy detector as a local spectrum sensing method for DSS, however in this case one needs to make the assumption that the noise level is the same at every node in the network, otherwise the threshold can not be set properly. In GoF based spectrum sensing, the threshold for the binary test depends only on the desired false alarm probability and not on the local noise powers. Motivated by this nice feature of GoF based spectrum sensing, we consider the goodness of fit (GoF) test statistic to be exchanged among cognitive radio (CR) users (consensus variable) instead of the energy. Moreover, a weighted consensus based DSS scheme is proposed and compared to the conventional consensus based on DSS. Simulations are conducted to show the effectiveness of the consensus algorithm based on GoF test.
\end{abstract}

Keywords-Cognitive Radio; Spectrum Sensing; Consensus Algorithms; Goodness of Fit test.

\section{INTRODUCTION}

Cognitive radio (CR) has emerged as a possible solution to improve spectrum utilization by allowing unlicensed (secondary) users to operate in the licensed band as long as the latter is absent, while avoiding interference to primary users [1]. To attain this goal, spectrum sensing is an indispensable task in cognitive radio, which enables the secondary users to detect the presence of a primary user (PU) [2] [3]. In practical applications, the received signal at each CR user may suffer from the hidden primary terminal problem and uncertainty due to fading and shadowing. In order to address the above issues, the cooperative spectrum sensing is proposed to enhance the detection performances by exploiting the spacial diversity of multiple CR users [9], [10].

Cooperative spectrum sensing can be performed in two models: centralized or distributed as illustrated in figure 1 . The former requires a common receiver (fusion center) to collect sensing results from all CR users in order to make final decision about the presence of a PU signal. However, a distributed scheme permits to CR users to share individual sensing results with their neighbours in order to make their own sensing decisions. This scheme is more suitable for cognitive radio ad-hoc networks (CRAHN), in which no hierarchical structure is involved, therefore, any node failure would not result in the failure of the entire network [5].

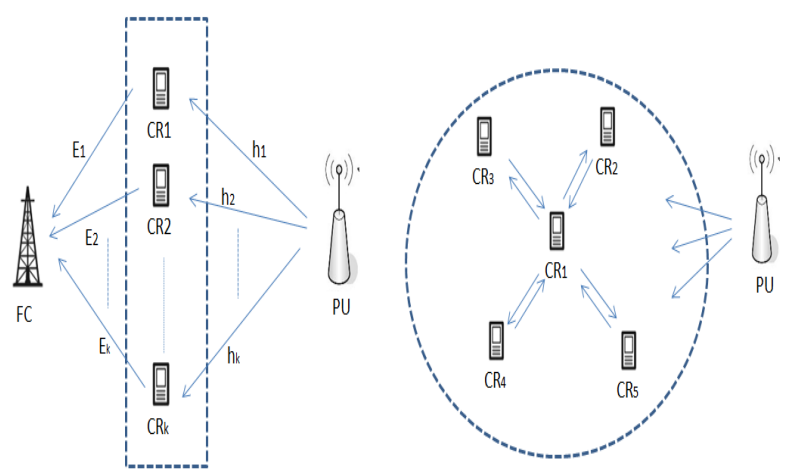

Fig. 1: Centralized Cooperative Spectrum Sensing (left) and Distributed Cooperative Spectrum Sensing (right)

A large number of studies have adopted a centralized cooperative spectrum sensing such [8] [7] [6], where a central unit (fusion center) collects hard or soft sensing information from cognitive radios, makes a final decision about the presence of PU, and broadcasts this information to other CR users.

Beside, distributed spectrum sensing has been a subject of several studies in recent years. Consensus algorithms have been utilized in order to obtain an agreement value in distributed systems [4].

In [11], a biologically inspired consensus-based spectrum sensing scheme without a fusion centre was proposed. A fully distributed spectrum sensing scheme is presented, where each CR user uses a biologically inspired computation rule to generate an updated state of the consensus variable. Authors in [12], extend the latter work with fixed bidirectional and random graphs. In the proposed scheme, CR users exchange messages based on local interaction without a centralized common receiver, and the consensus of the CR users is used to make the final decision. In [13], consensus-based spectrum sensing similar to that proposed in [12], is used in order to improve the security of CRAHNs using ID-based cryptography 
with threshold secret sharing. A weighted consensus-based spectrum sensing scheme is proposed in [15]. The CR users measure energy based on energy detection and then exchange the measured energy with its neighbours. The information exchanged is weighted according to its own estimated $S N R$ value. These algorithms perform detection in two time phases, one phase to take the measurement and an another phase to run the consensus algorithm. In [14], a distributed detection scheme based on diffusion strategies which can track changes in the PU state is proposed, i.e. a new measurement is incorporated into the algorithm on the fly. It is worth to mention that in the cited works, the assume that the noise is the same in every CR node.

In this paper, we aim to perform detection in a distributed way, i.e., without fusion center, relying on a new metric to be exchanged among CR users known as a GoF test statistic. In [18], it was shown that the GoF based spectrum sensing outperforms the conventional energy detection, moreover, the method is less sensitive to noise uncertainty [20] and the test statistic is independent of noise power [19]. Hence, the distributed consensus based on spectrum sensing is presented relying on the communication of GoF test statistic values among CR users. Moreover, a weighted consensus based DSS scheme is proposed and compared to the conventional consensus based DSS. It is shown that the proposed weighted consensus based DSS presents better performances, in terms of decision and efficient detection, compared to the conventional consensus based spectrum sensing.

The rest of the paper is as follows. Section II presents the network model for consensus algorithms. Section III explains the spectrum sensing model. Section IV involves the methodology adopted to develop a scheme for consensus based spectrum sensing based on GoF values. Section V presents the proposed weighted consensus algorithm for DSS using GoF based spectrum sensing. Section VI provides the simulation results and some discussion. Finally, conclusion is made in Section VI.

\section{NetWORK MODEL FOR Distributed SPECTRUM SENSING}

We model a cognitive radio network as a graph $G=(V, E)$ with N CR users collaborating to detect the presence or absence of a signal, where $\mathrm{V}$ is the vertices of the graph (identied by the index of the CRs $i=\{1, \ldots, N\}$ ) and $\mathrm{E}$ is the edges of the graph represented as the set of links between each pair of CRs. Links $(i, j)$ is denoted $e_{i j}$ and refers to the information flowing from vertex $\mathrm{j}$ to vertex $\mathrm{i}$ which is equivalent to information flowing from vertex $\mathrm{i}$ to vertex $\mathrm{j}$, if no direction is assigned to the edges (undirected graph). The set of all CR neighbours of a vertex $i$ is defined as $N_{i}=\left\{j \in V: e_{i j} \in E\right\}$. The maximum degree of the CRN is defined as the maximum number of neighbours of a $C R$ node.

The adjacency matrix $A$ of $G$ is the matrix with entries $a_{i j}$ given by

$$
a_{i j}= \begin{cases}1, & \text { if } e_{i j} \in E \\ 0 & \text { otherwise }\end{cases}
$$

We assume a bidirectional communication between any two CR users, i.e.; $a_{i j}=a_{j i}, \forall i, j \in N$. The Laplacian $L$ of the graph $G$ is defined as :

$$
l_{i j}= \begin{cases}\left|N_{i}\right|, & \text { if } j=i \\ -1, & \text { if } j \in N_{i} \\ 0 & \text { otherwise }\end{cases}
$$

The matrix $L$ is positive semi-definite.

\section{SPECTRUM SENSING MODEL}

The first stage of distributed spectrum sensing based on consensus scheme is a local measurement performed by each CR user. The statistic hypothesis test for local spectrum sensing can be modelled as:

$$
x_{i}(t)= \begin{cases}w_{i} & H_{0} \\ h_{i}(t) s(t)+w_{i} & H_{1}\end{cases}
$$

where $s(t)$ is the unknown signal of the primary user, $w_{i}(t)$ is the zero-mean additive white Gaussian receiver noise of the $i$ th CR user, and $h_{i}(t)$ is the channel gain from the primary user to the $i$ th CR user.

As mentioned before, on previous works [12] [11] [15], the distributed consensus based sensing schemes use energy detector as a local sensing method. The energy detection based spectrum sensing [16] consists of passing the received signal through a band-pass filter of bandwidth $W$ and center frequency $f_{s}$. The filtered signal then is squared and integrated over the sensing period $\mathrm{T}(\mathrm{m}=2 \mathrm{TW}, \mathrm{m}$ : sample size $)$. The output from integrator is distributed among neighbour CR users for consensus. $Y_{i}$ is formulated based $\mathrm{ED}$ as:

$$
Y_{i}=\sum_{k=1}^{m}\left|x_{i}(k)\right|^{2}, i=1,2, \ldots, N
$$

$Y_{i}$ in the above equation is the sum of the square of $m$ independent Gaussian distributed random variables. As a result, $Y_{i}$ follows the central chi-square distribution under hypothesis $H_{0}$, otherwise, $Y_{i}$ follows the non-central chi-square distribution.

Once the local sensing is performed, CR users communicate their local information with their neighbours until reaching the consensus. The local $\mathrm{CR}$ nodes can then take a decision based on this consensus value by comparing it to a threshold. The problem is that to set this threshold, one needs to know the noise power, however the noise could vary due to local interferences, differences in AGC setting, hardware impairments etc, so the threshold can not be set properly. The GoF based sensing requires only the knowledge of the noise distribution under $H_{0}$ hypothesis and the threshold for the binary test depends only on the desired false alarm probability and not the local noise power seen by the CR nodes.

As it is mentioned, in this paper, instead of communicating the energy, we propose communicate the GoF test statistic (for example: the Anderson Darling test) among CR users, which 
means that the local sensing is a GoF based spectrum sensing method.

GoF tests were proposed in mathematical statistics by measuring a distance between the empirical distribution of the observation made and the assumption distribution. In CRNs, the GoF test is used to solve a binary detection problem and decide whether the received samples are drawn from a distribution with a Cumulative Distribution Function (CDF) $F_{0}$, representing the noise distribution, or they are drawn from some distribution different from the noise distribution. The statistical hypothesis test is given by:

$$
\begin{gathered}
H_{0}: F_{m}(x)=F_{0}(x) \\
H_{1}: F_{m}(x) \neq F_{0}(x),
\end{gathered}
$$

for a random sample of $\mathrm{m}$ independent and identically distributed observations, where $F_{m}(x)$ is the empirical CDF of the received sample and can be calculated by:

$$
F_{m}(x)=\left|\left\{i: x_{i} \leq x, 1 \leq i \leq m\right\} / m\right|,
$$

where $|\bullet|$ indicates cardinality, $x_{1} \leq x_{2} \leq \ldots \leq x_{m}$ are the samples under test and $m$ represents the total number of samples.

There have been many goodness of fit test proposed in literature. The most important one is the Anderson-Darling test $A_{m, i}^{2}$. The expression of $A_{m, i}^{2}$ can be given according to [18] as:

$$
A_{m, i}^{2}=-m-\frac{\sum_{k=1}^{m}(2 k-1)\left(\ln z_{k}+\ln \left(1-z_{(m+1-k)}\right)\right)}{m}
$$

for $i=1, \ldots, N$ and with $z_{k}=F_{0}\left(x_{k}\right)$.

Each CR user exchanges the GoF test value $A_{m, i}^{2}$ with its neighbours, and then update it based on the received GoF test values from neighbours using consensus algorithms (details in the next section).

\section{The CONSEnsus Algorithms FOR Distributed SPECTRUM SENSING}

For the N CR users distributed according to the graph $G$, we assign them a set of states variable $x_{i}$ (consensus variable) for $i \in N$. The consensus algorithms aim to distribute the $x_{i}$ 's through an iterated process. By achieving consensus, the consensus variable $x_{i}$ progressively converges to the common value $x^{*}$ such as $x_{i}(k) \rightarrow x^{*}, k \rightarrow \infty$, where $k$ is the discrete time.

We distinguish different cases:

1 - It is said that the average consensus is achieved if all the individual state variable $x_{i}$, asymptotically converge to the average value : $x^{*}=\operatorname{Ave}(x)=\frac{1}{N} \sum_{i=1}^{N} x_{i}(0)$.

2 - It is said that the maximum consensus is achieved if all the individual state variable $x_{i}$, asymptotically converge to the maximum value $x^{*}=\max _{i=1}^{N} x_{i}(0)$.
3 - It is said that the minimum consensus is achieved if all the individual state variable $x_{i}$, asymptotically converge to the minimum value $x^{*}=\min _{i=1}^{N} x_{i}(0)$.

It is worth noting that the OR rule and the AND rule for cooperative spectrum sensing can be viewed as a form of maxconsensus and min-consensus respectively.

The performance of consensus algorithms is associated with the connectivity of CRN. The consensus based spectrum sensing algorithms can be expressed using a discrete-time state equation:

$$
x_{i}(k+1)=x_{i}(k)+\varepsilon \sum_{j \in N_{i}}\left(x_{j}(k)-x_{i}(k)\right)
$$

where $x_{i}(k)$ is the updated state at time $k$ of CR user $i, N_{i}$ denotes the neighbour set of CR user $i$ and $\varepsilon$ is a consensus parameter (step-size) satisfies:

$$
0<\varepsilon<\left(\underset{i}{\max }\left|N_{i}\right|\right)^{-1} \doteq 1 / \Delta
$$

where $\Delta$ is called the maximum degree of the network.

The consensus algorithm can be written in the vector form as:

$$
\left.x_{(} k+1\right)=P x(k)
$$

where $x=\left[x_{1}, \ldots, x_{N}\right]^{T}$ and $P=I-\epsilon L$ is called the Perron marix. $P$ is a stochastic matrix if the condition in (9) is ensured. Since $G$ is an indirected connected graph, therefore, $P$ is a doubly stochastic matrix, which means that $P$ is a non negative matrix and all of its row sums and column sums are equal to one.

In this paper, we construct $P$ based on the Metropolis weights [17] where the $p_{i j}$ are defined as:

$$
p_{i j}= \begin{cases}\frac{1}{1+\max \left(\left|N_{i}\right|,\left|N_{j}\right|\right)}, & \text { if }(i, j) \in E \\ 1-\sum_{j \in N_{i}}, & \text { if } i=j \\ 0 & \text { otherwise }\end{cases}
$$

In this alternative method, the knowledge of the maximum degree of the network is not needed. Since that any two neighbouring nodes exchange their degree.

There are two stages in the consensus spectrum sensing scheme.

In the first stage of spectrum sensing, every CR user performs GoF based spectrum sensing to get a local measurement $A_{m, i}^{2}$. We set up the initial GoF test vector such as: $x_{i}(0)=A_{m, i}^{2}$.

In the second stage, the average consensus algorithm or the maximum-consensus algorithm is conducted iteratively based on the fixed graph model at time $k=0,1,2, \ldots$. The iterative process is done until all the individual states $x_{i}(k)$ converge towards a common value $x^{*}$. Then, a decision is taken by every CR user by comparing the common value $x^{*}$ with a pre-defined threshold $\lambda$, every CR user obtains the global decision as: 


$$
\begin{aligned}
& H_{0}: x^{*}<\lambda \\
& H_{1}: x^{*} \geq \lambda
\end{aligned}
$$

$\lambda$ is chosen on the function of the predefined $P f a$ according to table 1 . The table 1 gives a corresponding $\lambda$ for some critical values of $P f a$ through Monte-Carlo simulations.

\section{Weighted Average Consensus for Distributed SPECTRUM SENSING}

In this section, motivated by [15], we present a weighted consensus for DSS. Compared to [15], our scheme use weights based on the local measured value by each CR node and its neighbours values (GoF test statistic). Moreover, the weights are updated at each step. In other terms, the weights are set according to the channel condition. Knowing that the GoF test statistic reflects the channel condition, we can use the GoF test statistic itself as a weight. Setting weights in the consensus algorithm favourites the CR nodes with higher values of GoF statistic measurement. The proposed weighted consensus is formulated as:

$$
x_{i}(k+1)=w_{i}(k) x_{i}(k)+\varepsilon \sum_{j \in N_{i}} w_{j}(k)\left(x_{j}(k)-x_{i}(k)\right)
$$

$$
\text { where: } w_{i}(k)=\frac{x_{i}(k)}{x_{i}(k)+\sum_{l \in N_{i}} x_{l}(k)} \text { and } w_{j}(k)=
$$

$$
\begin{aligned}
& \frac{x_{j}(k)}{x_{i}(k)+\sum_{l \in N_{i}} x_{l}(k)} \\
& \quad \text { with } w_{i}(k)+\sum_{j \in\left(N_{i}\right)} w_{j}(k)=1
\end{aligned}
$$

For convenience, we re-write the equation (13) in the following compact form:

$$
x(k+1)=P_{w} x(k)
$$

where $x=\left[x_{1}, \ldots, x_{N}\right]$ and $P_{w}$ is the weighted Perron matrix.

It can be shown that by using (13) the consensus value $x^{*}$ will converge to the weighted average of initial GoF test statistic values $\frac{1}{N} \sum_{i=1}^{N} w_{i}(0) x_{i}(0)$. This convergence is concluded from the famous Perron Frobenius Theorem [21].

Likewise, each CR user performs sensing based on GoF based detector, and simultaneously collects GoF test statistics from its connecting neighbour CR users. It then updates its sensing value ( $\mathrm{GoF}$ statistic) iteratively using its own and neighbours sensing information according to the algorithm (13). As time elapses, the sensing information will be diffused through the network and finally each $\mathrm{CR}$ user obtains a consensus value $x^{*}$. Every CR user is able to take the global decision by comparing the common value $x^{*}$ with a predefined threshold $\lambda$ such as:

\begin{tabular}{|l|c|c|c|c|}
\hline Consensus based & Pfa & 0.1 & 0.05 & 0.01 \\
DSS & Threshold & 1.180 & 1.249 & 1.348 \\
\hline Weighted consensus & Pfa & 0.1 & 0.05 & 0.01 \\
based DSS & Threshold & 2.036 & 2.217 & 2.710 \\
\hline
\end{tabular}

TABLE I: Threshold values for some given $P f a$

$$
\begin{aligned}
& H_{0}: x^{*}<\lambda \\
& H_{1}: x^{*} \geq \lambda
\end{aligned}
$$

The value of $\lambda$ is determined for a specific value of $P f a$. A table listing values of $\lambda$ corresponding to different false alarm probabilities can be computed by Monte Carlo approach. The table 1 gives a corresponding $\lambda$ for some critical values of Pfa.

\section{Simulation RESUltS}

In this section, we conduct simulation to study the performances of the proposed weighted consensus scheme. We show the convergence of the weighted scheme and evaluate the detection performance of the weighted consensus based DSS through Monte Carlo simulation. The simulations are done such that, each $\mathrm{CR}$ user has different $S N R$ values varying randomly from $-20 d B$ to $0 d B$. We consider a network topology with 50 nodes as depicted in figure 2 .

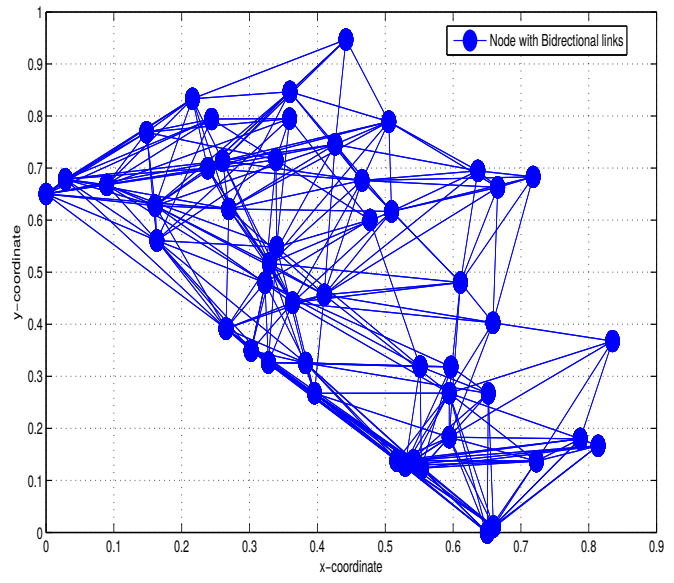

Fig. 2: The network with $50 \mathrm{CR}$ users and fixed graph

Figure 3 shows the convergence of the GoF statistic calculated based on the received signal from a PU in the network. As one can observe, despite the fact that the initially sensed measurement varies greatly due to their different wireless channel conditions for different CR users, a consensus is achieved after several iterations. The same goes for the proposed weighted scheme, when we show that the GoF test values, calculated initially by different $\mathrm{CR}$ users, tend to converge towards a consensus value after several iterations. It is observed that the consensus value, reached by the proposed weighted consensus, is higher than the consensus value reached by the conventional consensus. 


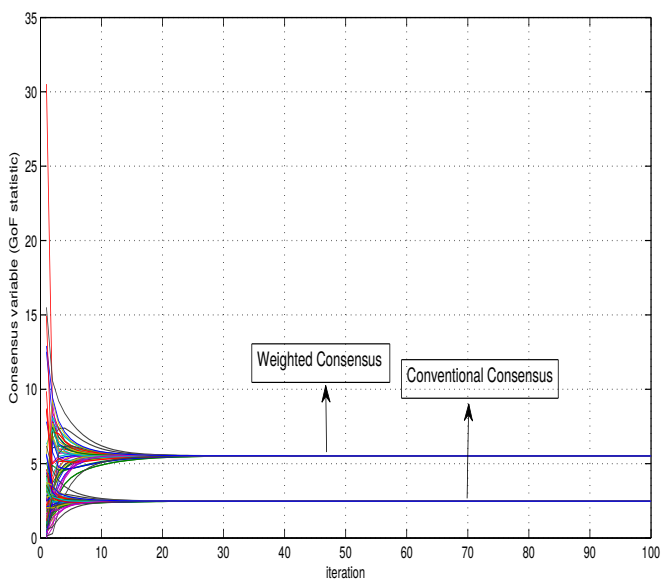

Fig. 3: Convergence of the network for conventional consensus based GoF test

In figure 4, we plot the ROC curves (detection probability versus false alarm probability) for the proposed weighted consensus based DSS and the conventional consensus based DSS under AWGN channel with CRN size 50 nodes. From figure 4 , it is shown that the proposed weighted consensus detection based on GoF test have a significant improvement compared the conventional consensus detection based on GOF, in terms of detection.

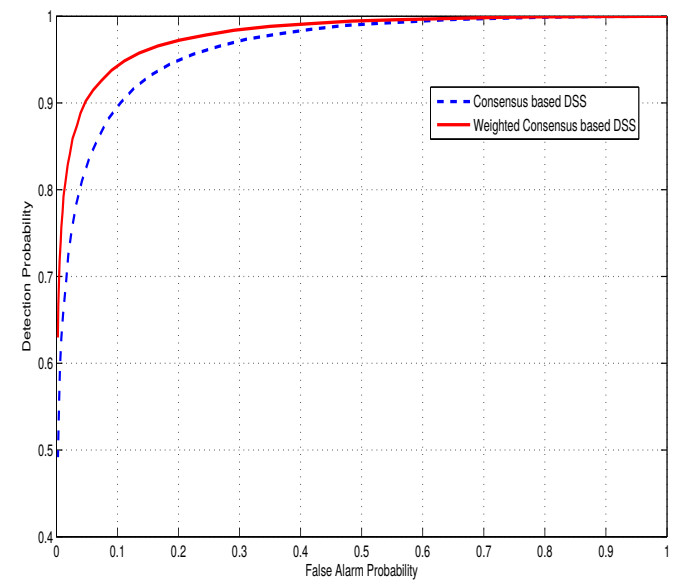

Fig. 4: Detection probability versus false alarm probability for proposed weighted consensus based DSS using GoF for local sensing

\section{CONCLUSION}

In this paper, we have presented a distributed spectrum sensing, based on consensus algorithms. The detection problem is modelled as a graph networking topology. We proposed to use a GoF test as a local spectrum sensing, because this test statistic is independent of the local noise power seen by the $\mathrm{CR}$ nodes and then considering a GoF test statistic to be exchanging among $\mathrm{CR}$ users to reach consensus. In addition, a weighted consensus based DSS is proposed. The performance of the proposed method is studied and compared to the conventional consensus scheme, based on GoF as local spectrum sensing. We showed that the proposed method outperforms the conventional one in terms of detection performances.

\section{REFERENCES}

[1] J. Mitola. "Cognitive radio" An integrated agent architecture for software defined radio, Phd dissertation. Phd thesis, MIT, 2000.

[2] J. Ma, G. Li, and B. H. Juang, "Signal processing in cognitive radio," Proceedings of the IEEE, vol. 97, no. 5, pp. 805-823, 2009.

[3] T. Yucek and H. Arslan, "A survey of spectrum sensing algorithms for cognitive radio applications," IEEE Communications Surveys and Tutorials, vol. 11, no. 1, pp. 116-130, Quat. 2009.

[4] R. Olfati-Saber, J. A. Fax, and R. M. Murray, "Consensus and cooperation in networked multi-agent systems," Proc. of the IEEE, vol. 95, no. 1, pp. 215-233, Jan. 2007.

[5] I. F. Akyildis, W.-Y. Lee and K. R. Chowdhury, "CRAHN: cognitive radio ad hoc networks" AD HOC NETWORKS, vol. 7, no. 5, pp.810836, July 2009.

[6] G. Ganesan, T.J. Lim, Cooperative Spectrum Sensing in Cognitive Radio networks, IEEE, Dynamic Spectrum Access Networks (DySPAN), Nov 2005.

[7] E. Visotsky, S. Kuffner, R. Peterson, On Collaborative Detection of TV Transmissions in support of Dynamic Spectrum Sharing, IEEE, Dynamic Spectrum Access Networks (DySPAN), Nov 2005.

[8] A. Ghasemi, E. S. Sousa, Collaborative spectrum sensing for opportunistic access in fading environments, IEEE, Dynamic Spectrum Access Networks (DySPAN), Nov 2005.

[9] K.Ben Letaief and Z. Wei, "Cooperative Communication for Cognitive Radio Networks," Proceedings of the IEEE, vol. 97, pp. 878-893, 2009

[10] W. Haijun, et al., " Cooperative Spectrum Sensing in Cognitive Radio under Noise Uncertainty," IEEE 71st Vehicular Technology Conference, pp. 1-5, 2010.

[11] F.R. Yu, M. Huang, H. Tang, "Biological inspired consensus-based spectrum sensing in mobile ad hoc networks with cognitive radios," IEEE Netw. 24 (3) (2010) 2630.

[12] Z. Li, F.R. Yu, M. Huang, "A distributed consensus-based cooperative spectrum sensing scheme in cognitive radios," IEEE Trans. Veh. Technol. 59 (1) (2010) 383393.

[13] H. Tang, F.R. Yu, M. Huang, Z. Li, "Distributed consensus-based security mechanisms in cognitive radio mobile ad hoc networks," IET Commun. 6 (8) (2012) 974983.

[14] F. S. Cattivelli, A. H. Sayed, " Distributed Detection Over Adaptive Networks Using Diffusion Adaptation," IEEE Transactions On Signal Processing, Vol. 59, NO. 5, May 2011.

[15] W. Zhang, Z. Wang, Yi. Guo, H. Liu, Y. Chen, J. Mitola III, "Distributed cooperative spectrum sensing based on weighted average consensus," in: Proc. of IEEE Global telecommunication conference (GLOBECOM), December 2011, pp. 16.

[16] F. F. Digham, M. S. Alouini, and M. k. Simon. "On the energy detection of unknown signals over fading channels. IEEE Trans. on Communications, 55(1);21-24, 2007.

[17] L. Xiao, S. Boyd, and S. Lall, " A scheme for robust distributed sensor fusion based on average consensus," in Proc. Fourth International Symposium on Information Processing in Sensor Networks, pp. 63-70, 2005.

[18] D. Teguig, V. Le Nir and B. Scheers : 'Spectrum sensing method based on goodness of fit test using chi-square distribution', Electronics Letters, Volume 50, Issue 9, 24 April 2014, p. 713715.

[19] T. W. Anderson, D. A. Darling: ' Asymptotic Theory of certain Goodness of Fit Criteria Based on Stochastic Process," Annals of Mathematical Statistic, Vol. 23, pp.193-212, 1952.

[20] D. Teguig, V. Le Nir B. Scheers and F. Horlin : 'Spectrum sensing Method Based on the Likelihood Ratio Goodness of Fit test under noise uncertainty', IJERT, Vol. 3, Issue 9, September 2014.

[21] M. Carl, " Matrix Analysis and Applied Linear Algebra". SIAM, 2000. 